\title{
Automated Sample Preparation for Environmentally Reactive Materials Systems
}

\author{
R. R. Cerchiara, P. E. Fischione, M. F. Boccabella and A. C. Robins \\ E. A. Fischione Instruments, Inc., 9003 Corporate Circle, Export, PA 15632
}

Application of automated sample preparation to materials systems of various compositions and configurations has yielded benefits for field emission scanning electron microscopy (FESEM). The first group to be studied included microelectronics with Silicon ( $\mathrm{Si}$ ) based transistor technologies and $\mathrm{SiO}_{2}$ based dielectrics. [1] The metallic components included $\mathrm{Al}$ and $\mathrm{Cu}$ and the refractory elements (W, Ta) present within via liners and interconnects. The second group included microelectronics with a low - $\mathrm{k}$ dielectric added as an interlayer within the $\mathrm{SiO}_{2}$ for enhanced performance. [2] The third group included microelectronics with retained packaging consisting of engineered polymers. [3] After embedding within a suitable epoxy, a cross-section was prepared to reveal its internal microstructure as well as external components (e.g. ball bonds, brazes). This and a fourth group to be processed included nanoscale devices based on $<45 \mathrm{~nm}$ transistor technologies. $[3,4]$ With these material systems, the metal gates depended on complex Ti aluminides, oxides and nitrides deposited during the fabrication process.

The standard protocol for any materials system is initiated with mechanical sectioning. This is followed by pre-smoothing of the cut or cleaved surface by mechanical grinding and polishing. The polished surface is subjected to a combination of processes including argon - oxygen plasma cleaning (PC), ion beam etching (IBE), reactive ion etching (RIE), and ion beam sputter coating (IBSC). Plasma cleaning effectively removes hydrocarbons, while ion beam etching removes native oxides and reduces surface topography to the nanometer level. Reactive ion etching selectively removes a certain phase to produce differential contrast in high resolution imaging modes which depend on secondary electron emission in the FESEM. Deposition of thin $(<4 \mathrm{~nm})$ and amorphous layers of carbon or metal (Ir, Cr, W) promote the emission of SE's and stabilize the electrical properties of the etched surface. As environmentally reactive materials are prepared, the maintenance of vacuum level and minimizing of residual gas is critical as sequences are executed within a single process chamber. Improvements in imaging and analysis will be reviewed for reactive material systems, including those observed with electron back scatter diffraction (EBSD).

\section{References}

[1] R. R. Cerchiara, P. E. Fischione, J. J. Gronsky, W. F. Hein, D. D. Martin, J. M. Matesa, A. C. Robins and D. W. Smith, "Recent Developments in Automated Sample Preparation for FESEM", Proceedings of the $29^{\text {th }}$ International Symposium for Testing and Failure Analysis, Santa Clara, CA, Nov. 2-6, 2003, pp. 288 - 300.

[2] R. R. Cerchiara, H. A. Cook, P. E. Fischione, A. C. Robins, D. W. Smith, E. R. Beach, C .J. Wood, S. J. Rozeveld and J. Waeterloos, "Automated Sample Preparation of Low k Dielectrics for FESEM", Proceedings of the $31^{\text {th }}$ International Symposium for Testing and Failure Analysis, Nov. 6-10, 2005.

[3] R. R. Cerchiara, P. E. Fischione, M.F. Boccabella and A. C. Robins, "Automated Sample Preparation of Packaged Microelectronics for FESEM", Proceedings of the $34^{\text {th }}$ International Symposium for Testing and Failure Analysis, Nov. 2-6, 2008.

[4] R. R. Cerchiara, P. E. Fischione, M.F. Boccabella and A. C. Robins, "Automated Sample Preparation of Nanoscale Devices for FESEM”, Microscopy and Microanalysis 2009, Richmond, VA, July 2009. 